\title{
Future of nuclear parton densities
}

\author{
Néstor Armesto* \\ Departamento de Física de Partículas and IGFAE, \\ Universidade de Santiago de Compostela, 15782 Santiago de Compostela, Galicia-Spain \\ E-mail: nestor.armesto@usc.es
}

\begin{abstract}
Parton densities PDFs are most important objects both from a fundamental point of view, for characterising the partonic content of hadrons and nuclei, and for the application of collinear factorisation in hadronic and nuclear collisions. Nuclear parton densities nPDFs are greatly unknown, compared to those in the proton, due to the scarcity of experimental data. In this contribution I will first review the present status of nPDFs. Then I will discuss the perspectives of further determining them in presently existing experiments, particularly in $\mathrm{pPb}$ collisions at the LHC. Finally I will examine the possibilities that planned experiments, both hadronic machines - HL-LHC and electron-nucleus colliders - EIC, LHeC and FCC-eh, offer for constraining nPDFs.
\end{abstract}

XXVI International Workshop on Deep-Inelastic Scattering and Related Subjects (DIS2018) 16-20 April 2018

Kobe, Japan

${ }^{*}$ Speaker. 


\section{Introduction}

Collinear parton densities (PDFs) are the universal pieces that provide the initial flux of partons to compute particle production in hard processes involving hadrons [1]. Therefore, they are basic objects for the calculation of such cross sections. Assuming that collinear factorisation holds for collisions involving nuclei, the standard way to deal with the experimental fact that nuclear structure functions cannot be described by the superposition of non-interacting protons and neutrons $[2,3]$ is absorbing the nuclear effects in a modification of parton densities in the nuclear medium nPDFs. Then, the techniques of DGLAP evolution equations and global fits used to extract proton PDFs can be used to extract nPDFs [4]. The feasibility of such extraction and the compatibility of the predictions of collinear factorisation using existing nPDFs with new sets of data test the factorisation hypothesis, see the most recent sets of nPDFs in $[5,6,7,8,9,10]$.

On top of their intrinsic interest for the partonic characterisation of nuclei, nPDFs are required to compute observables in heavy-ion collisions. With the goal of the heavy-ion program being the characterisation of the hot dense medium produced in the collisions, the existing uncertainties in nPDFs reflect in sizeable uncertainties in the extraction of medium parameters, see e.g. Fig. 84 in [11]. It is therefore of key importance to reduce such uncertainties. At present, the experimental possibilities come from the proton-nucleus programmes [12] and the study of ultraperipheral collisions UPCs [13] at RHIC and the LHC. In the future, electron-ion colliders: the EIC in the US [14] and the LHeC/FCC-eh at CERN [15], will be the machines for a precise determination of nPDFs.

Here I will review the present status of nPDFs and the possibilities for further determining them in existing and future experimental facilities. Due to limitations of space, I apologise in advance for the large amount of material that I will skip. I will mainly discuss the standard nuclear modification factor in nucleus $A$ for the PDF $f_{i}^{A}\left(x, Q^{2}\right)$ of any parton species $i$, defined as $R_{i}^{A}\left(x, Q^{2}\right)=f_{i}^{A}\left(x, Q^{2}\right) /\left[A f_{i}^{p}\left(x, Q^{2}\right)\right]$.

\section{Present status of nuclear parton densities}

Presently (see e.g. [16]), nPDFs are poorly constrained due to two facts. On the one hand, data come from a large variety of nuclei and the number of data points for any of them, particularly for $\mathrm{Pb}$ for which it is less than 50 coming from fixed target DIS and DY experiments and from $\mathrm{pPb}$ collisions at the LHC, is very small compared to proton analyses. This fact makes the fit for a single nucleus impossible and the modelling of the $A$-dependence of the parameters in the initial conditions mandatory. On the other hand, the region covered by existing data, see Fig. 1, is very small compared to that for the proton. The most up to date analyses include between 1000 and 2000 data points for 14 nuclei and are performed at next-to-leading accuracy $[5,6,7,8,10]$ (even there exists some preliminary attempt at next-to-next-to-leading [9]). Differences between them mainly arise from the different sets of data included in the analysis and from the different functional forms employed for the initial conditions to be fitted. As a result, all parton species are very weakly constrained at small $x<10^{-2}$, gluons at large $x>0.2$, and flavour decomposition is largely unknown - a natural fact for $u$ and $d$ due to the approximate isospin symmetry in nuclei. The impact of presently available LHC data, studied in [10], is quite modest with some constrains on the gluon in the region $0.01<x<0.3$. 

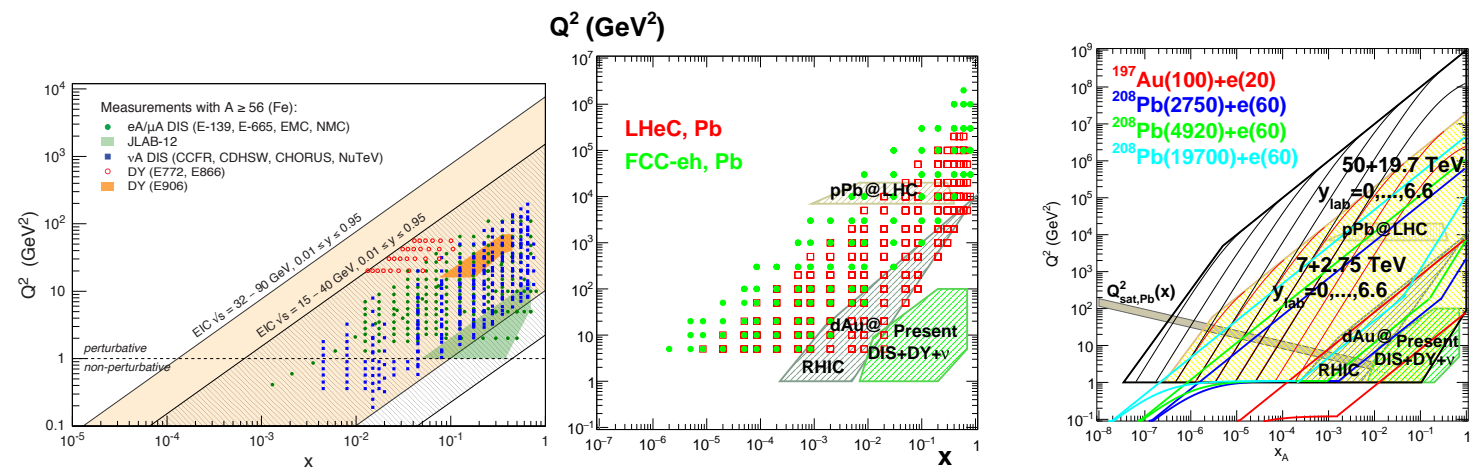

Figure 1: Regions in the $x-Q^{2}$ kinematic plane covered in electron-nucleus collisions by the EIC (left) [17], the LHeC/FCC-eh (middle) [18] and a comparison between the regions covered by electron-nucleus and present and future pA colliders (right) [18]. The region covered by presently available experimental data is also shown in each plot.

\section{Future of the determination of parton densities}

\subsection{Kinematics}

The kinematic regions in the $x-Q^{2}$ plane studied in present and future experiments are shown in Fig. 1. It can be observed that: (i) the presently available region in constrained to $x>0.01$ and, except for $\mathrm{LHC} \mathrm{pPb}$ data, to $Q^{2}<100 \mathrm{GeV}^{2}$; (ii) electron-ion colliders offer an extension of the presently available region that can be as large as 3 to 4 orders of magnitude in $x$ and $Q^{2}$; (iii) pA colliders offer further enlargement (an analogous region can be drawn for UPCs, see [13]). While hadronic colliders are unbeatable in terms of kinematics, DIS offers a cleaner experimental setup with fully reconstructed kinematics and more first-principle theoretical calculations available.

\subsection{Constraints from the (HL-)LHC}

There exist several observables at the LHC that offer constraints on nPDFs:

- Dijet data in $\mathrm{pPb}$ collisions constrain the nuclear glue for $x>0.01[19,20]$.

- Data on EW boson production in $\mathrm{pPb}$ constrain the difference between $u$ and $d$ quarks [21].

- Exclusive $\mathrm{J} / \psi$ production in UPCs constrains the nuclear glue at small $x$ [22].

- Quarkonium and open heavy flavours in $\mathrm{pPb}$ constrain the nuclear glue at small $x$ [23] (Fig. 2).

In future Runs 3 and 4 at the LHC, larger statistics can be expected for several observables like EW bosons and bottomonium in $\mathrm{pPb}$, and UPCs, and new observables like inclusive and diffractive dijets in UPCs or tops in $\mathrm{pPb}$ [24]. And theoretical and experimental work is demanded to reduce the large uncertainties that exist on quarkonium and open heavy flavour production in UPCs and $\mathrm{pPb}$ and to clarify the relevance of collective phenomena at small transverse momenta [25, 26], that would preclude the use of collinear factorisation.

\subsection{Constraints from electron-ion colliders}

DIS is the ideal place to determine PDFs through the measurement of both inclusive quantities like reduced cross sections and structure functions, and more exclusive quantities like those with 

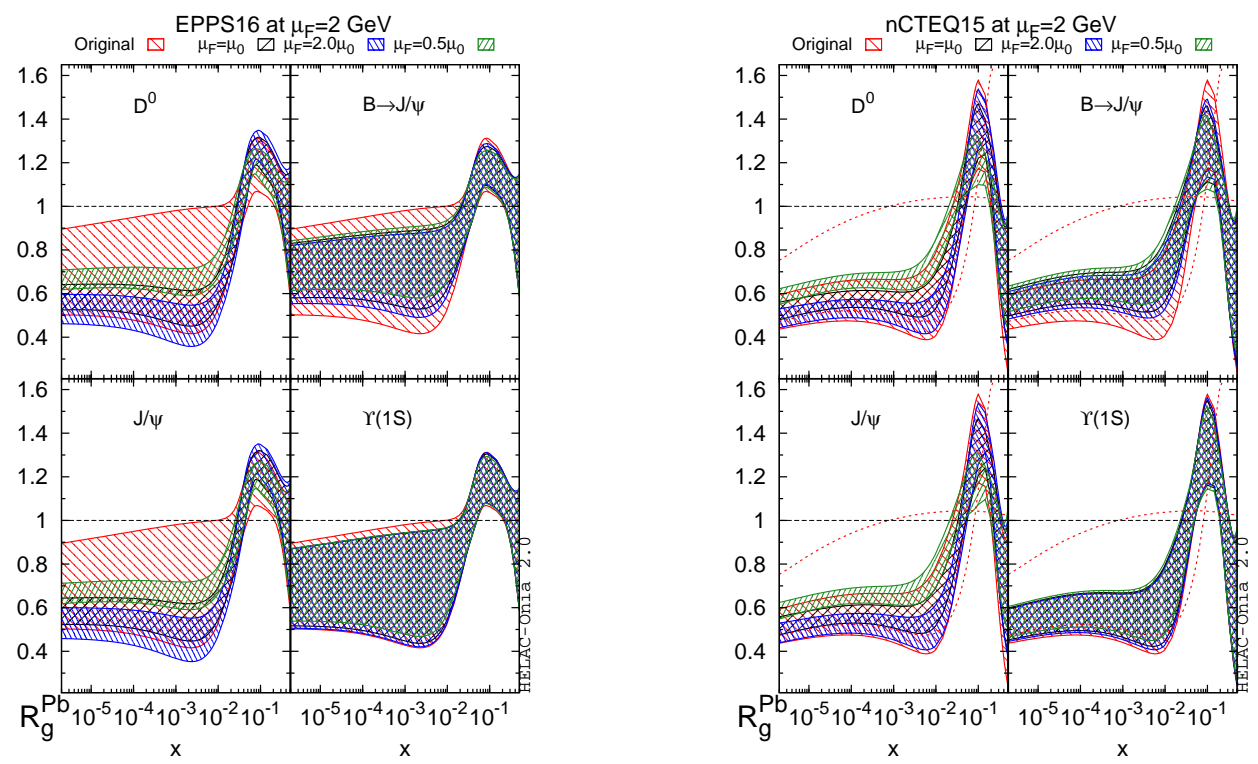

Figure 2: Impact of quarkonium and open heavy flavour production in $\mathrm{pPb}$ collisions at the LHC on the nuclear modification factor for the glue, using reweighting techniques on the EPPS16 (left) and nCTEQ15 (right) nPDF sets [23].

identification of flavours. Here I offer two recent examples of the expected impact of measurements at future eA colliders:

- At the EIC [17], substantial constrains on the nuclear glue are expected for $x>10^{-3}$ from fits to reduced cross sections, and additional constrains at large $x$ from charm measurements, see Fig 3 .

- At the LHeC/FCC-eh, similar studies were performed in the past in the same EPPS16 [10] framework, see [27]. Here, in Fig. 4 I show the result of a fit [18] using xFitter [28] that should reflect the ultimate precision that can be achieved in terms of determination of nuclear parton densities at the $\mathrm{LHeC}$ and the FCC-eh.

From the comparison with Fig. 2, the much larger constraining power of eA compared to hadronic collisions is evident.

In conclusion, I have briefly discussed the present status of nPDFs and the future opportunities for determining them both at the (HL-)LHC and at future eA colliders, EIC and LHeC/FCC-eh.

Acknowledgments: I thank the organisers for their invitation to provide this talk. I acknowledge financial support by Ministerio de Ciencia e Innovación of Spain under projects FPA2014-58293C2-1-P, FPA2017-83814-P and Unidad de Excelencia María de Maetzu under project MDM-20160692, Xunta de Galicia (Consellería de Educación) within the Strategic Unit AGRUP2015/11, and FEDER. This work has been performed in the framework of COST Action CA15213 THOR.

\section{References}

[1] J. C. Collins, D. E. Soper and G. F. Sterman, Adv. Ser. Direct. High Energy Phys. 5 (1989) 1 [hep-ph/0409313]. 

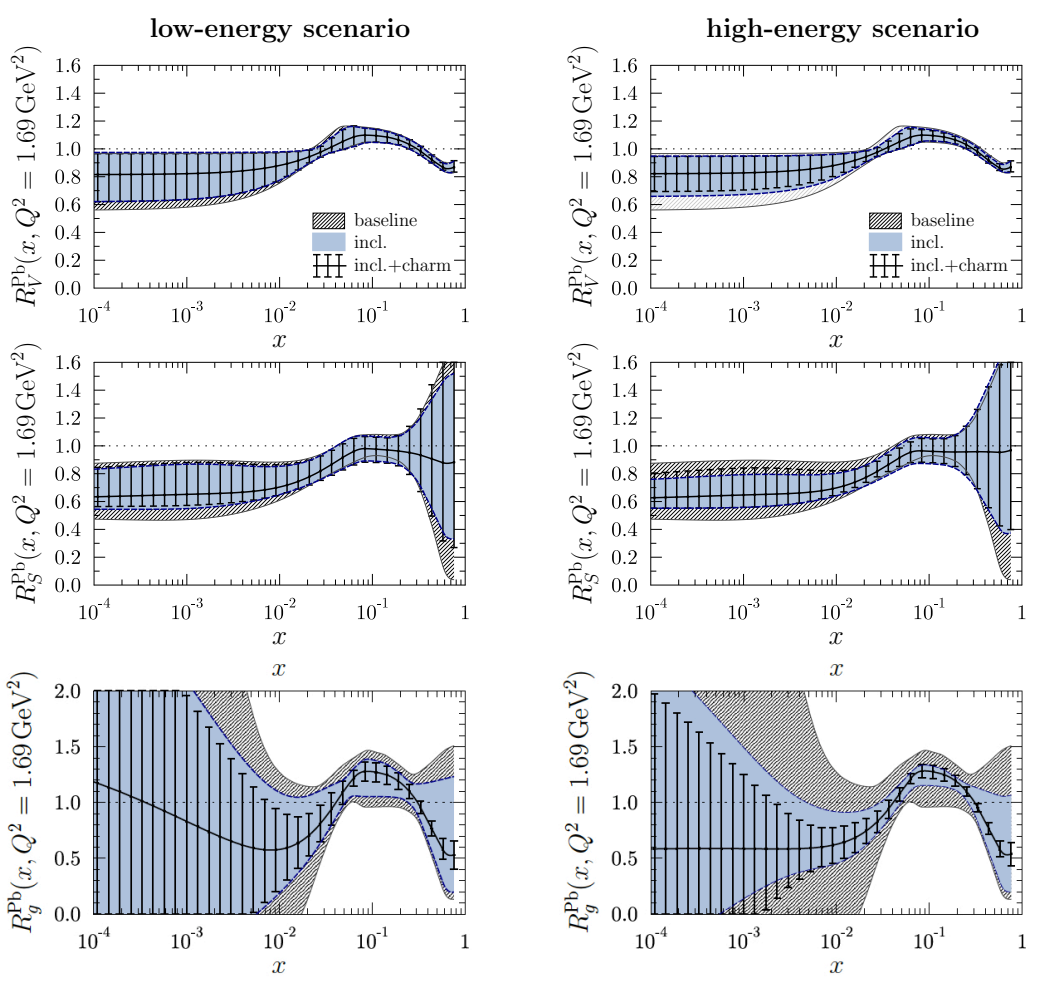

Figure 3: Constraints on the nuclear modification factor for the average valence (top), average sea (middle) and glue in $\mathrm{Pb}$ using inclusive (blue band) and inclusive+charm (error bars) data [17], compared with the original EPPS16 uncertainties (grey band), for low (left) and high (right) energy EIC scenarios.

[2] M. Arneodo, Phys. Rept. 240 (1994) 301.

[3] D. F. Geesaman, K. Saito and A. W. Thomas, Ann. Rev. Nucl. Part. Sci. 45 (1995) 337.

[4] K. J. Eskola, Nucl. Phys. B 400 (1993) 240.

[5] M. Hirai, S. Kumano and T.-H. Nagai, Phys. Rev. C 76 (2007) 065207 [arXiv:0709.3038 [hep-ph]].

[6] K. J. Eskola, H. Paukkunen and C. A. Salgado, JHEP 0904 (2009) 065 [arXiv:0902.4154 [hep-ph]].

[7] D. de Florian, R. Sassot, P. Zurita and M. Stratmann, Phys. Rev. D 85 (2012) 074028 [arXiv:1112.6324 [hep-ph]].

[8] K. Kovarik et al., Phys. Rev. D 93 (2016) no.8, 085037 [arXiv:1509.00792 [hep-ph]].

[9] H. Khanpour and S. Atashbar Tehrani, Phys. Rev. D 93 (2016) no.1, 014026 [arXiv:1601.00939 [hep-ph]].

[10] K. J. Eskola, P. Paakkinen, H. Paukkunen and C. A. Salgado, Eur. Phys. J. C 77 (2017) no.3, 163 [arXiv:1612.05741 [hep-ph]].

[11] A. Andronic et al., Eur. Phys. J. C 76 (2016) no.3, 107 [arXiv:1506.03981 [nucl-ex]].

[12] C. A. Salgado et al., J. Phys. G 39 (2012) 015010 [arXiv:1105.3919 [hep-ph]].

[13] A. J. Baltz et al., Phys. Rept. 458 (2008) 1 [arXiv:0706.3356 [nucl-ex]]. 

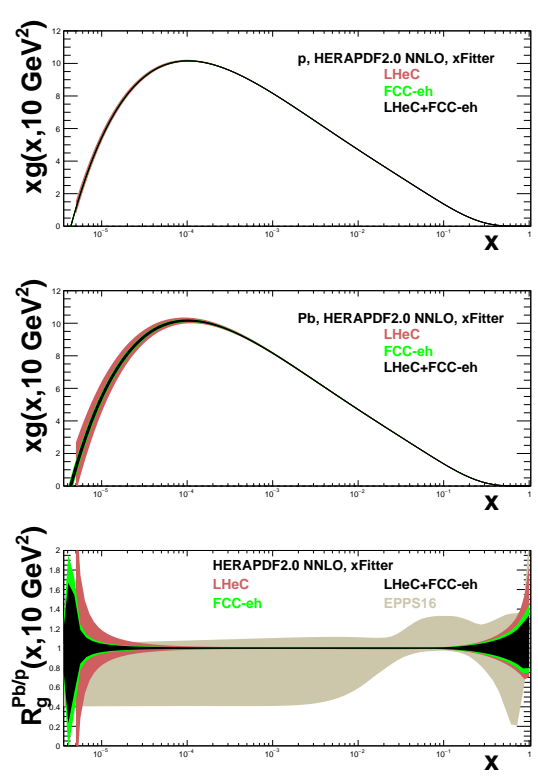
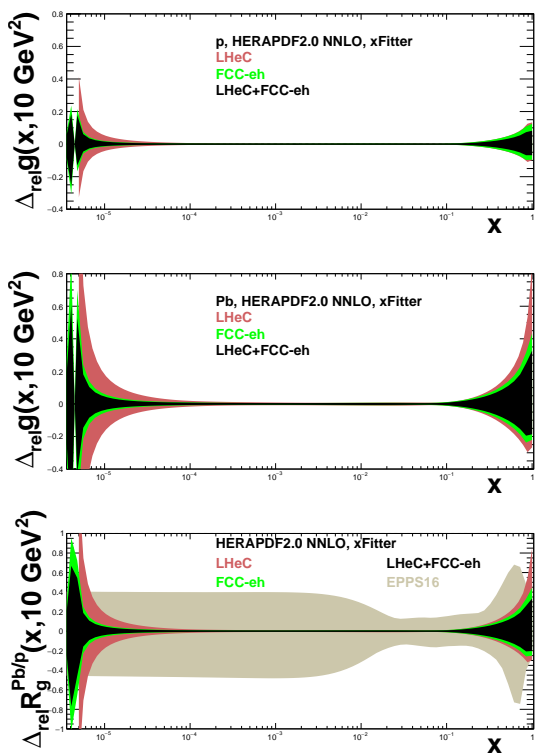

Figure 4: Proton (top left) and $\mathrm{Pb}$ (middle left) gluon PDFs (per nucleon) and their ratio (bottom left), and their respective uncertainties (right plots) at the LHeC, the FCC-eh and their combination, extracted [18] using xFitter. The plots at the bottom show the EPPS16 results for comparison with the present situation (see the legends on the plots for the meaning of the colours).

[14] A. Accardi et al., Eur. Phys. J. A 52 (2016) no.9, 268 [arXiv:1212.1701 [nucl-ex]].

[15] J. L. Abelleira Fernandez et al. [LHeC Study Group], J. Phys. G 39 (2012) 075001 [arXiv:1206.2913 [physics.acc-ph]].

[16] N. Armesto, EPJ Web of Conferences 171 (2018) 11001.

[17] E. C. Aschenauer, S. Fazio, M. A. C. Lamont, H. Paukkunen and P. Zurita, Phys. Rev. D 96 (2017) no.11, 114005 [arXiv:1708.05654 [nucl-ex]].

[18] N. Armesto, talk at the 2nd FCC Physics Workshop, CERN, January 15-19 2018, https://indico.cern.ch/event/618254/contributions/2833211/.

[19] A. M. Sirunyan et al. [CMS Collaboration], arXiv:1805.04736 [hep-ex].

[20] K. J. Eskola, P. Paakkinen and H. Paukkunen, arXiv:1806.08208 [hep-ph].

[21] A. Kusina et al., Eur. Phys. J. C 77 (2017) no.7, 488 [arXiv:1610.02925 [nucl-th]].

[22] V. Guzey and M. Zhalov, JHEP 1310 (2013) 207 [arXiv:1307.4526 [hep-ph]].

[23] A. Kusina, J. P. Lansberg, I. Schienbein and H. S. Shao, arXiv:1712.07024 [hep-ph].

[24] D. d'Enterria, K. Krajczár and H. Paukkunen, Phys. Lett. B 746 (2015) 64 [arXiv:1501.05879 [hep-ph]].

[25] A. M. Sirunyan et al. [CMS Collaboration], Phys. Rev. Lett. [arXiv:1804.09767 [hep-ex]].

[26] S. Acharya et al. [ALICE Collaboration], arXiv:1805.04367 [nucl-ex].

[27] H. Paukkunen [LHeC study Group], PoS DIS 2017 (2018) 109 [arXiv:1709.08342 [hep-ph]].

[28] S. Alekhin et al., Eur. Phys. J. C 75 (2015) no.7, 304 [arXiv:1410.4412 [hep-ph]]. 